Typ-2-Diabetes

\title{
Hypoglykämien sind ein häufiger Grund für schlechte Therapietreue
}

- Leichte Unterzuckerungen sind bei Typ2-Diabetikern häufig und ein Grund für schlechte Therapietreue. Die Patienten riskieren dadurch vermeidbare Langzeitkomplikationen. Diese Erkenntnisse resultieren aus der GAPP2-Studie, einer globalen Online-Befragung von ca. 3000 Diabetikern und 1600 Diabetologen, über die Dr. Martin Grundner, Frankfurt/M., berichtete.

$80 \%$ der Patienten gaben an, schon einmal Unterzuckerungen gehabt zu haben, bei $36 \%$ war dies im letzten Monat der Fall. Nahezu die Hälfte der Patienten berichtete, schon einmal eine Basalinsulin-Dosis ausgelassen zu haben, in den meisten Fällen mit Absicht. Viele Patienten spritzen Insulin auch zeitversetzt oder verringern ihre Insulindosis. Häufigstes Motiv für diese Dosisunregelmäßigkeiten war die Reduktion des Hypoglykämierisikos. Viele Patienten gaben auch an, dass es sie belaste, das Insulin immer zur gleichen Zeit injizieren zu müssen.

Auffällig waren die Ergebnisse aus Deutschland: Hier war generell die Angst vor Hypoglykämien ausgeprägt, insbesondere vor Unterzuckerungen im Schlaf oder beim Autofahren. Grundner's Rat: Der Arzt sollte Patienten aktiv auf Hypoglykämien ansprechen.

Schwere Hypoglykämien sind einer der häufigsten Gründe für Kliniknotaufnahmen. Sie erhöhen die Sterblichkeit sowie die Risiken für Demenz und Herzinfarkt, berichtete Dr. Andreas Liebl, Bad Heilbrunn. Moderne Insuline können laut Liebl dazu beitragen, Hypoglykämien zu vermeiden: „Detemir ist das Langzeitinsulin mit der geringsten Wirkungsschwankungsbreite von Tag zu Tag, einem wichtigen Risikofaktor für Hypoglykämien. Zudem führen Hypo- glykämien unter Detemir-Behandlung früh zu Beschwerden wie Schwitzen, sodass der Patient frühzeitig gewarnt wird."

Kürzlich erhielt mit Degludec (Tresiba ${ }^{\circledR}$ ) ein neues, besonders lange wirksames Basalinsulin die europäische Zulassung. Die Halbwertszeit ist mit 24 Stunden doppelt so lang wie das die etablierten Basalinsuline. Daraus resultiert eine besonders gleichmäßige Wirkung, das Risiko für nächtliche Hypoglykämien ist um $26 \%$ niedriger als bei Insulin Glargin, so Liebl. Er hält Degludec für eine wichtige Bereicherung, die v.a. für Patienten mit Hypoglykämien und solche geeignet ist, die unzuverlässig spritzen.

- Dr. med. Dirk Einecke

Quelle: Grünwalder Gespräche „Den Diabetes jederzeit im Griff haben", München/Grünwald, Februar 2013 (Veranstalter: Novo Nordisk)

\section{Einfach aufsprühen}

\section{Hämoglobinspray versorgt chronische Wunden mit Sauerstoff}

- Die Hypoxie kann zur Verlangsamung oder Stagnation der Wundheilung führen, da viele Wundheilungsprozesse von einer ausreichenden Sauerstoffzufuhr abhängen. Einen innovativen Ansatz zur Hypoxieüberwindung bei chronischen Wunden stellt ein seit April 2012 als Medizinprodukt der Klasse III zugelassenes HämoglobinSpray (Granulox ${ }^{\oplus}$ ) dar. Das Spray enthält natürliches, wasserlösliches Hämoglobin, das Umgebungssauerstoff bindet und ihn zum Wundgrund transportiert. Es wird nach der Wundreinigung auf die Wunde gesprüht, danach folgt wie gewohnt das Abdecken der Wunde mit einer hydroaktiven Auflage. Durch die lokale Oxygenierung werden die Neubildung von Granulationsgewebe, die Epithelisierung und der Aufbau eines stabilen Narbengewebes gefördert. „Das Hämoglobin-Spray sollte von
Anfang an adjuvant zur Kausaltherapie der Primärerkrankung zum Einsatz kommen, um eine Chronifizierung der Wunde zu verhindern. Die Anwendung lässt sich unkompliziert in den Pflegealltag integrieren", so Michael Sander, Geschäftsführer der SastoMed GmbH, Georgsmarienhütte.

Prof. Petr Arenberger, Prag, präsentierte die aktuellen Ergebnisse einer von ihm geleiteten prospektiven, einfach-verblindeten, randomisierten und placebokontrollierten klinischen Studie an Patienten mit Ulcus cruris venosum (Der Hausarzt 2013; 2: 54). Über einen Zeitraum von 13 Wochen erhielten jeweils 36 Probanden eine topische Behandlung mit dem Spray bzw. mit einer 0,9\%-igen hämoglobinfreien Kochsalzlösung. In der Verumgruppe konnte eine deutliche Reduktion der Wundgrößen um durchschnittlich 53\% ge-

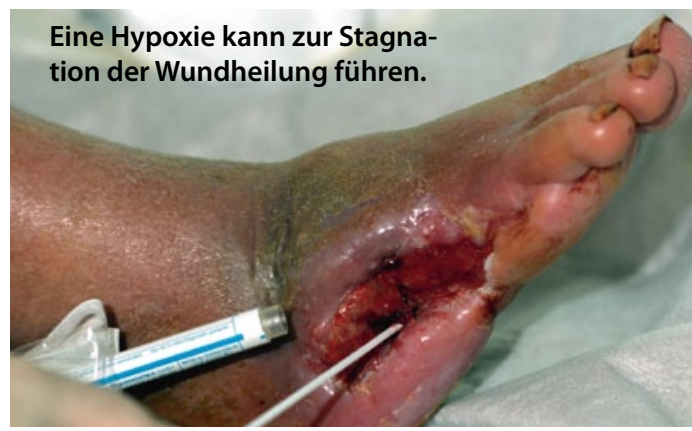

genüber der Kontrollgruppe dokumentiert werden. Auch das Wundschmerzempfinden verringerte sich in der Verumgruppe kontinuierlich, während es in der Kontrollgruppe konstant hoch blieb. „Das Hämoglobin-Spray ist eine einfache und wirksame Therapieergänzung ohne zu beobachtende Nebenwirkungen", resümierte Arenberger.

\footnotetext{
- Gudrun Girrbach

Quellen: Satellitensymposium „Wirksame Therapie zur Hypoxieüberwindung chronischer Wunden", Interdisziplinären WundCongresses (IWC), Köln, November 2012 (Veranstalter: SastoMed)
} 